\title{
Recent strategies targeting HIV glycans in vaccine design
}

\author{
Satoru Horiya, lain S MacPherson \& Isaac J Krauss ${ }^{\star}$
}

\begin{abstract}
Although efforts to develop a vaccine against HIV have so far met with little success, recent studies of HIV-positive patients with strongly neutralizing sera have shown that the human immune system is capable of producing potent and broadly neutralizing antibodies (bnAbs), some of which neutralize up to $90 \%$ of HIV strains. These antibodies bind conserved vulnerable sites on the viral envelope glycoprotein gp120, and identification of these sites has provided exciting clues about the design of potentially effective vaccines. Carbohydrates have a key role in this field, as a large fraction of bnAbs bind carbohydrates or combinations of carbohydrate and peptide elements on gp120. Additionally, carbohydrates partially mask some peptide surfaces recognized by bnAbs. The use of engineered glycoproteins and other glycostructures as vaccines to elicit antibodies with broad neutralizing activity is therefore a key area of interest in HIV vaccine design.
\end{abstract}

Although antiretroviral drugs have greatly improved the survival of HIV patients, the high cost of these drugs, together with the emergence of resistance, make a preventative vaccine the most attractive long-term solution to the global pandemic. Most vaccines designed to elicit a neutralizing antibody response have comprised HIV envelope proteins gp120 and/or gp41 and have fallen short of stimulating antibodies with either enough potency or breadth to neutralize the diverse HIV strains present in nature ${ }^{1}$. However, extensive study of HIV-positive individuals has recently provided a wealth of data about potent bnAbs, which naturally arise in some infected individuals $^{2-10}$. It is now increasingly clear that many of these bnAbs bind epitopes on gp120 partly or exclusively composed of oligosaccharide moieties (glycans) ${ }^{11-26}$. Moreover, in the case of bnAbs that bind purely peptide epitopes, such as the CD4-binding site, there is evidence that certain glycans sterically mask this region and impede recognition by germline antibodies necessary for initiation of a bnAb response $\mathrm{e}^{27,28}$. In this review, we will describe recent HIV vaccine design strategies that exploit this knowledge, either through production of glycosylated antigens that mimic the epitopes of bnAbs or through engineered glycoprotein fragments lacking certain masking glycans.

\section{BnAbs as templates for vaccine design}

The typical antibody response to HIV or to recombinant monomeric gp120 glycoprotein is unable to neutralize diverse HIV strains for several reasons ${ }^{29-33}$. Non-neutralizing antibodies bind surfaces that are accessible only on monomeric gp120 that has detached from the viral surface, and thus they cannot bind and neutralize the virus itself. These same binding surfaces are inaccessible on the intact gp120 trimers that remain on the viral membrane (Fig. 1a). Other antibodies can bind trimeric gp120 on the virus but target nonconserved parts of the glycoprotein; these antibodies are neutralizing but strain specific. By contrast, each bnAb targets a conserved surface that is accessible on the trimer and provides clues as to which viral surfaces are vulnerable for neutralization ${ }^{2}$. If the epitope of a bnAb (the surface it binds to) can be determined, this information can serve as the basis for vaccine design. In principle, structures that precisely mimic the bn Ab epitope but lack the other viral glycoprotein elements could be useful as vaccines because antibodies generated against these mimetic constructs should be focused on the bn Ab epitope and thus neutralize in a broad manner, similarly to the template bnAb. Though this logic is appealing, in practice there are several challenges. First, for bnAbs that bind carbohydrate epitopes, the heterogeneity of HIV glycosylation can make it difficult to precisely define the structures that comprise the epitope. Moreover, epitopes may be composed of several glycans or peptide fragments that are not continuous in the HIV polypeptide sequence and are thus difficult to mimic with small designed peptides or glycopeptides. Finally, even if one can design structural mimics of an epitope that are highly antigenic (recognized as tightly by the bnAb as is the natural epitope on the viral glycoprotein), they may not be immunogenic, i.e., they may not necessarily elicit an immune response. Moreover, if they are immunogenic, the resulting antibodies may still not bind the desired portion of the epitope mimic or to the viral epitope and may not neutralize the virus. Nevertheless, highly antigenic structures are desirable, if not sufficient, in vaccine design.

\section{Targeting specific germline antibodies}

Although antigens designed simply to bind a bnAb may fail to reelicit that bnAb in immunizations, there has been some speculation that the desired bn $\mathrm{Ab}$ response is more likely if the antigen can also bind the germline precursor of the desired bnAb ${ }^{34,35}$. Germline antibodies are the repertoire of lower-affinity, broader-specificity antibodies already present in an individual before infection that are direct products of VDJ recombination, with no additional mutations. After germline B cells are activated in response to a pathogen, the affinity maturation process leads to evolution of B-cell mutants bearing higher-affinity 'mature' antibodies. During this process, bnAbs thus acquire many unusual features, such as long complementarity-determining region loops, framework mutations and even domain exchange. Putative germline Abs (gl-Abs) corresponding to many bnAbs have been predicted through bioinformatic methods, and in most cases they exhibit little or no binding to HIV envelope proteins ${ }^{9,36-38}$. It is possible that HIV has evolved such that its conserved epitopes avoid recognition by the human germline antibody repertoire, which might explain why immunogens that simply mimic the bnAb epitope do not necessarily stimulate the desired bnAb response. Carbohydrates apparently have a role in masking conserved epitopes from gl-bnAb recognition, as it has been shown that deletion of certain glycans from gp120 rescues its ability to be recognized by germline antibodies. We will discuss this 'glycan deletion' strategy ${ }^{27,28}$ in further detail later in this review. 

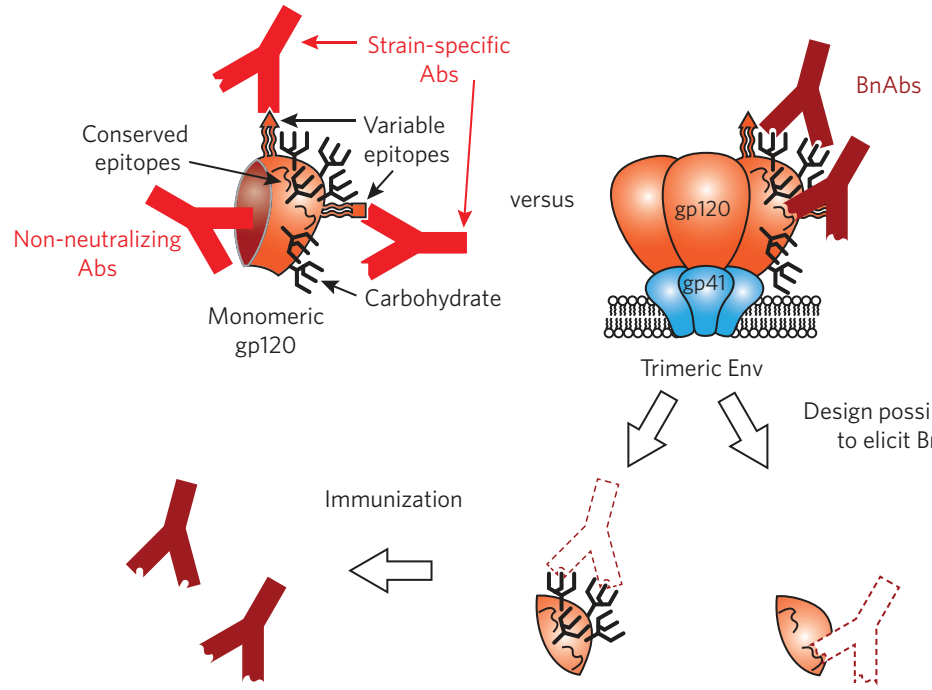

Immunization

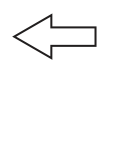

bnAb-like response

Conserved carbohydrate or glycopeptide epitopes

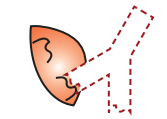

Unmasked conserved peptide epitopes

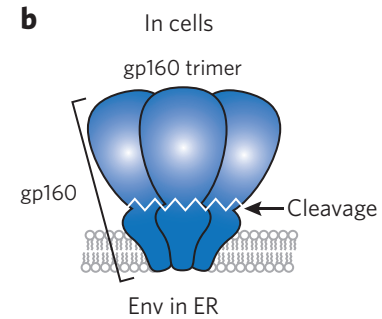

Env in ER

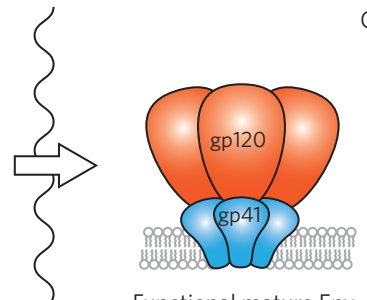

Functional mature Env
On virus

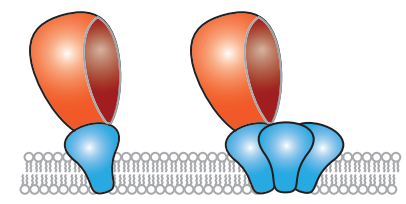

'Junk' Env

Figure 1 | The trimeric structure of HIV Env and implications for antibody neutralization. (a) Nonneutralizing and strain-specific antibodies and bnAbs. BnAbs only bind conserved epitopes located on the gp120 trimeric assembly. Immunogens displaying these conserved epitopes may be able to focus the immune response on these structures and thus elicit antibodies with broadly neutralizing specificity. (b) Proteolytic processing of Env. In infected cells, the Env is synthesized as the precursor protein gp160, which undergoes signal peptide cleavage, folding, trimerization and glycosylation in the endoplasmic reticulum ${ }^{94-96}$. The resulting gp160 trimer is transported to the Golgi apparatus and cleaved into gp120 and gp41 subunits by furin-like proteases ${ }^{97-100}$. The mature gp120-gp41 trimer is unstable so that most virions contain 'junk Env' structures bearing exposed gp41 or gp120 monomers ${ }^{40}$.

\section{Structure and glycobiology of the HIV envelope}

HIV envelope protein structure. The functional envelope spike protein of HIV (Env) is a $\mathrm{C}_{3}$-symmetric assembly of three gp120 proteins, each noncovalently associated with an underlying transmembrane gp41 protein (Fig. 1b) ${ }^{39}$. Gp120 is responsible for recognition of the CD4 receptor on T cells, and gp41 is responsible for the mechanics of fusing the viral and host membranes. This trimer of heterodimers is unstable so that even the native HIV particles contain 'junk' Env proteins that have fallen apart at the gp120-gp120 or gp120-gp41 interfaces ${ }^{40}$. Monomeric gp41 and gp120 on the viral surface or in the bloodstream present many highly immunogenic surfaces that are not exposed in viral trimeric Env; they are thus problematic as immunogens as they elicit primarily antibodies that bind monomeric protein but not the functional trimer located on the virus (Fig. 1a,b). As an alternative, stable and soluble trimeric envelope constructs have been produced by engineering of gp160, the biosynthetic precursor protein, which is cleaved to form gp120 and gp41. These trimeric proteins, for example, 'SOSIP-gp140', have been produced by truncating the transmembrane domain of the gp41 sequence, and adding disulfides and other elements to stabilize gp41-gp120 and trimeric interfaces ${ }^{41}$. These constructs may be useful as immunogens because both their exposed peptide surface

and their glycosylation pattern may better mimic viral Env.

HIV N-linked glycosylation. Gp120 is extremely unusual in that its polypeptide sequence contains $\sim 25$ potential sites of $\mathrm{N}$-linked glycosylation ${ }^{42-47}$ (PNGs, amino acid sequence NXT/S), whereas typical mammalian glycoproteins contain no more than a few ${ }^{48,49}$. The glycans, which comprise about $50 \%$ the total gp 120 mass, confer two benefits on the virus. First, the mannose residues within these glycans bind the DC-SIGN receptor on dendritic cells, enabling the virus to accumulate on the surface of these cells and facilitating its presentation to $\mathrm{CD}^{+} \mathrm{T}$ cells for infection ${ }^{50-52}$. Second, the dense canopy of glycans sterically masks the underlying polypeptide surface against interaction with potentially neutralizing antibodies and is thus sometimes referred to as the 'glycan shield'. Because these glycans are functionally necessary, they are therefore somewhat conserved, and thus antibodies that bind them-for example, bnAbs $2 \mathrm{G} 12$ (refs. 4,11,12,15,6), PG9/16 (refs. 7,13,24) and the PGTs ${ }^{14,19,21-23,25,26}$ - may neutralize a broad range of viral strains, with up to $80 \%$ of circulating strains neutralized by a single antibody. Most importantly, some of these antibodies have been shown to exhibit a protective effect in vivo ${ }^{53-56}$. To design vaccines that may elicit 2G12-like or PG9-like antibodies, several groups have worked on the design of carbohydrate clusters that mimic the epitopes of these bnAbs (described below) ${ }^{57-81}$.

Ideally, the design of glycostructures to mimic HIV glycopeptide epitopes should be informed by detailed knowledge about the glycosylation of gp120. However, precise characterization of HIV glycosylation is very difficult, both because analytical methods are insufficient and because samples are heterogenous and/or difficult to produce with native glycosylation patterns. For instance, X-ray crystallography is hampered by glycosylation heterogeneity and by the difficulty of crystallizing glycoproteins. Therefore, crystallography is normally performed on samples that have been produced under non-native conditions to obtain homogeneous glycoforms and/or with samples whose glycans have been trimmed by glycosidases.

Gp120 glycosylation not only is heterogeneous but also depends on the HIV strain, the stage of HIV infection ${ }^{82}$, the producing cell type as well as the oligomeric form, i.e., whether the protein is obtained as recombinant cell-secreted gp120 monomer or gp140 trimer or from pseudovirus or actual viral particles ${ }^{83,84}$. For example, MS of the total glycans cleaved off the trimeric gp120 from viral particles indicates that the majority of glycans are high-mannosetype glycans (i.e., $62-79 \% \mathrm{Man}_{5-9} \mathrm{GlcNAc}_{2}$ ), with only the remaining 21-38\% being 'complex' or 'hybrid-type' glycans (Fig. 2a). By contrast, the same analysis of recombinant monomeric gp120 indicates a much higher percentage (up to 71\%) of complex and hybrid-type glycans. This difference could hypothetically arise from steric effects in the glycan-processing pathway (Fig. 2b); as gp120 is processed in the Golgi, glycans located in the most densely glycosylated regions of gp120 or in regions near the trimer interface are inaccessible to 
the glycosidases responsible for processing the immature high-mannose glycoforms into the more mature complex and hybrid types.

Although overall glycan content can be deduced from analysis of glycans cleaved from whole viral trimeric gp120 protein, determination of the specific distribution of which glycoforms are present at each site requires MS analysis of enzymatically digested gp120 (refs. 42-47). Although several studies have reported this type of analysis with samples of recombinant gp120 monomer, the most relevant glycosylation pattern must be obtained from analysis of gp120 trimers isolated from actual virus samples. It is challenging to obtain

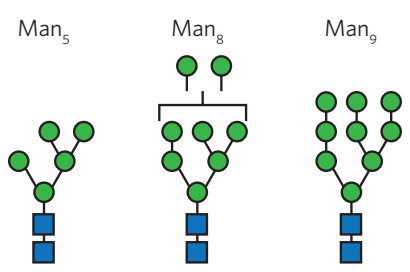

Oligomannose

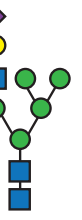

Hybrid sufficient quantities of this material for analysis, and no such studies have yet been reported. Therefore, the identity and position of glycans comprising the various bnAb epitopes has been only partially deduced from a combination of techniques. These include (i) mutation to delete various PNG sites, followed by observation of reduced bnAb binding, (ii) observation of antibody binding to specific glycans in glycan array or saturation transfer difference (STD) NMR studies and (iii) X-ray crystallography of bnAbs crystallized with glycans or Env constructs. Because of glycan heterogeneity and conformational flexibility, crystallographic studies of bnAb-Env complexes have typically used Env constructs treated with glycosidases and/or those produced under conditions that yield a simplified glycosylation profile. In light of data from several studies, an HIV glycosylation model was proposed in which gp120 can be divided into three different types of regions (Fig. 3) ${ }^{57,58}$. First, regions of gp120 close to the trimer interface are sterically accessible to glycosidases when gp120 remains monomeric but are inaccessible to glycosidases in trimeric gp120. Thus, glycans in this region remain as high-mannose types in mature gp120 trimers from virus-derived samples but are hybrid or complex types in secreted gp120 monomer isolated from cell cultures. A second important region of gp120 is the 'intrinsic high-mannose patch', exemplified by glycans in the vicinity of N332, N334, N392 and N295, in which the spatial density of glycosylation sites within a single gp120 protomer sterically impedes glycan processing even without gp120 oligomerization. Glycans in this region are recognized by bnAbs such as 2G12, PGT128, 121 and 135 (ref. 21). The remaining regions of gp120, which lie outside this densely glycosylated region and away from the trimer interface, are thought to bear more diverse and more extensively processed hybrid- or complex-type glycoforms, which may still comprise part of bnAb epitopes.

\section{G12-targeted vaccine design}

$2 \mathrm{G} 12$, isolated from the serum of an HIVpositive individual ${ }^{85}$, was the second antibody reported to have broad neutralization activity (half-maximum effective concentration $<50 \mu \mathrm{g} \mathrm{ml}^{-1}$ for $\sim 32 \%$ of HIV isolates tested) and the first reported to bind carbohydrates on gp120. Alanine scanning mutagenesis of gp 120 to delete PNG sites, together with glycosidase digestion experiments, showed that $2 \mathrm{G} 12$ binds a cluster of two to four high-mannose $\left(\mathrm{Man}_{8} \mathrm{GlcNAc}_{2}\right.$ and/or $\mathrm{Man}_{9} \mathrm{GlcNAc}_{2}$ ) glycans, most likely at a subset of positions N295, N339, N332 and N392 ${ }^{15,16}$. X-ray crystallography of 2G12 cocrystallized with $\mathrm{Man}_{9} \mathrm{GlcNAc}_{2}$ directly showed 2G12 interacting with three crystallographically unique copies of the glycan. These data were combined with a crystal structure of deglycosylated gp120 to create a model of the 2G12-gp120 interaction (Fig. 4) ${ }^{11,12}$. The primary binding contacts involve the nonreducing terminal four mannose units of the D1 arm, and $\mathrm{Man}_{4}$ or $\mathrm{Man}_{9}$ fragment glycans therefore bind 2G12 with affinity similar to that of $\mathrm{Man}_{9} \mathrm{GlcNAc}_{2}$. Moreover, this structure revealed that $2 \mathrm{G} 12$ has a unique domain-exchanged structure in which 


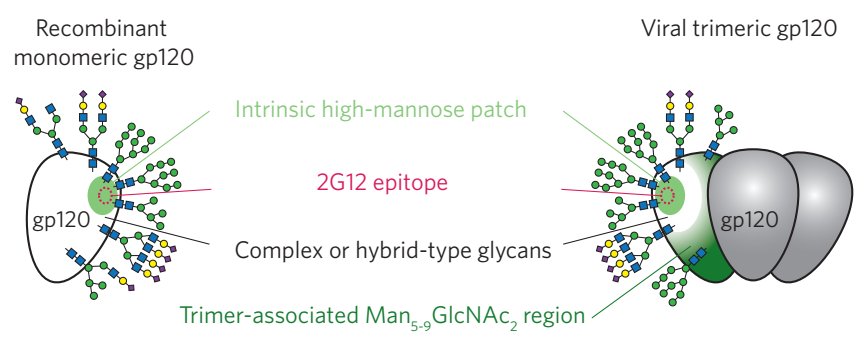

Figure 3 | Glycosylation profile of monomeric versus trimeric gp120. The glycosylation of monomeric recombinant gp120 differs from that of trimeric gp120 on the viral membrane, with the latter containing a much higher percentage of high-mannose glycans. It has been proposed ${ }^{83,84}$ that high mannose content is greatest in the most sterically congested areas, including the interfaces between gp120 protomers in the trimer, and an intrinsic high-mannose patch within the monomer, where the density of PNG sites is particularly high. Glycans pictured in this figure (Consortium for Functional Genomics notation) are meant to represent classes of glycoforms found in each region rather than specific glycans located at each position.

the $V_{H}$ subunit of each antibody arm is paired with the $V_{L}$ domain of the other, bringing the two antibody arms together to form a single, large antigen recognition surface. 2G12 is thus capable of binding many tightly packed mannose residues together, resulting in a high avidity interaction. Whereas $\mathrm{Man}_{9} \mathrm{GlcNAc}_{2}$ itself binds $2 \mathrm{G} 12$ with a $K_{\mathrm{d}}$ of $180 \mu \mathrm{M}$, its multivalent presentation on gp120 results in a $K_{\mathrm{d}}$ of $\sim 5 \mathrm{nM}$, a 36,000-fold enhancement of recognition. Moreover, the special structure of $2 \mathrm{G} 12$ is essential: mutagenesis of $2 \mathrm{G} 12$ to eliminate the domain exchange feature virtually eliminates binding to gp120 (refs. 86,87).

On the basis of this information, many groups have worked to design multivalent oligomannose clusters that might mimic the 2 G12 epitope for use as vaccines to stimulate a 2G12-like antibody response. These oligomannose constructs can be evaluated in terms of two criteria: (i) antigenicity, i.e., their binding affinity for $2 \mathrm{G} 12$, and (ii) immunogenicity, i.e., their ability to stimulate a 2G12-like antibody response in vivo. Although high antigenicity is desirable

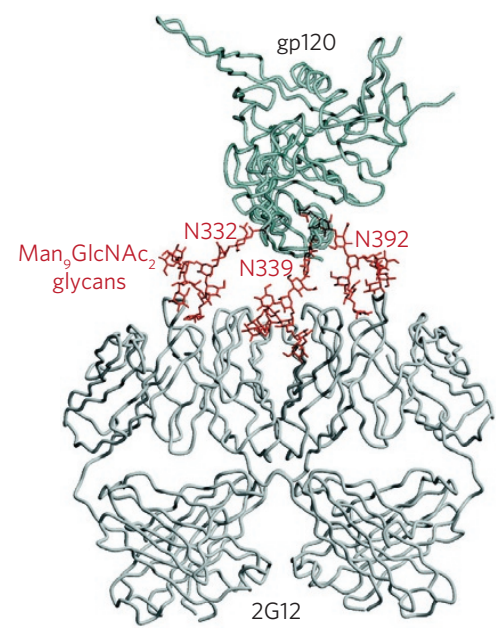

Figure 4 | Model of $\mathbf{2 G 1 2}$ glycan recognition of gp120. Image was taken from ref. 12, with permission. This model was created on the basis of the crystal structure of $2 \mathrm{G} 12 \mathrm{Fab}$ dimer with four $\mathrm{Man}_{9} \mathrm{GICNAc}_{2}$ moieties and alanine-scanning mutagenesis of gp120 to determine which PNG sites were required for $2 \mathrm{G} 12$ recognition ${ }^{16}$. The reducing termini of three glycans from the $2 \mathrm{G} 12-\mathrm{Man}_{9} \mathrm{GlcNAc}_{2}$ cocrystal structure were conformationally modeled to match Asn residues at positions N332, N339 and N392 in a separate, deglycosylated gp120 crystal structure.
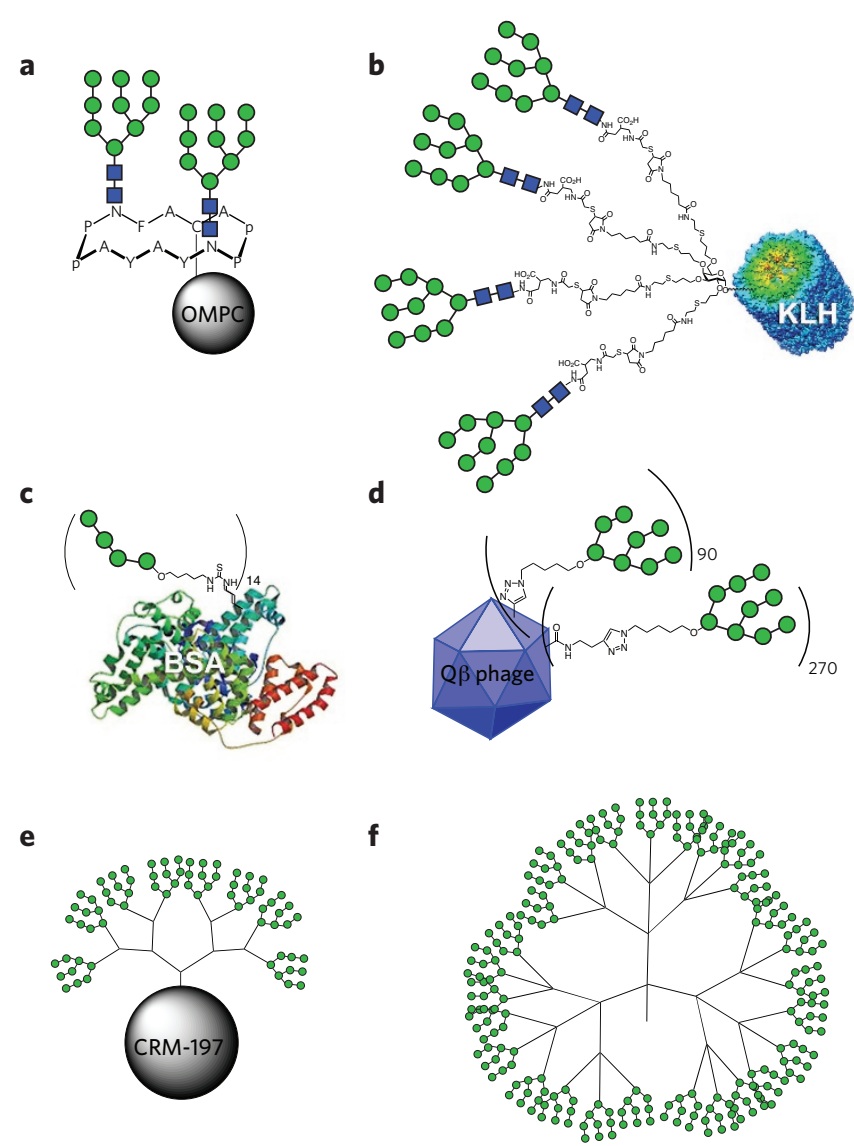

Figure 5 | Selected multivalent high-mannose glycan cluster antigens.

(a) Divalent cyclic peptide bearing two $\mathrm{Man}_{9} \mathrm{GlcNAc}_{2}$ glycans, connected by short natural Asn linkages, conjugated ( 2,000 copies) to outer membrane protein complex (OMPC) of Nesseiria meningitides carrier protein ${ }^{57,58}$. (b) Tetravalent $\mathrm{Man}_{9} \mathrm{GlcNAc}_{2}$ clustered via a flexible linker on galactose scaffold, conjugated to keyhole limpet hemocyanin (KLH) carrier protein $^{63}$. (c) Man $_{4}$-conjugated (14 copies) via short linker to BSA carrier protein $^{70}$. (d) Heteromultivalent clustering of $\mathrm{Man}_{8}$ or $\mathrm{Man}_{9}$ on $\mathrm{Q} \beta$ phage particles $^{71}$. (e) PAMAM $_{8}-M_{9}$, dendrimers conjugated (two copies) to CRM-197 carrier protein (Diphtheria toxoid) ${ }^{68}$. (f) Highly multivalent Man ${ }_{9}$ dendrimers ${ }^{67}$. In general, $2 \mathrm{G} 12 K_{\mathrm{d}}$ values are not reported, but constructs in a-e exhibit 2G12 affinities generally 50- to 1,000-fold weaker than that of gp120. Immunogenicity studies of constructs shown in $\mathbf{a}, \mathbf{b}, \mathbf{d}$ and $\mathbf{e}$ all reported mannose-binding antibody responses, though gp120 binding activity was weak or absent in animal sera. In contrast to the constructs in a-e, construct $\mathbf{f}$ is reported to be highly antigenic, with a $2 \mathrm{G} 12 K_{\mathrm{d}}$ of $\sim 3 \mathrm{nM}$, similar to that of gp120; however, no immunogenicity data have been reported for this construct.

in an epitope mimic, it is not necessarily sufficient to guarantee the desired immunogenicity; one does not really know whether an antigen is a good immunogen until it has been tested in animal immunogenicity studies.

Figure 5 summarizes the antigenicity and immunogenicity of representative $2 \mathrm{G} 12$ epitope mimics, which will only be only briefly discussed here, as they have been reviewed in detail elsewhere ${ }^{88}$. Diverse studies have reported multivalent clusters of high-mannose glycans attached to rationally designed peptide ${ }^{57-61}$, carbohydrate $^{62,63}$, steroid ${ }^{64}$, peptide nucleic acid ${ }^{65,66}$, dendrimer ${ }^{67,68}$ and gold nanoparticle ${ }^{69}$ backbones as well as on biomacromolecules such as $\mathrm{BSA}$ protein ${ }^{70}$ and Q $\beta$ phage particles ${ }^{71,72}$. In most cases, the reported 2 G12 affinity of these constructs has been 50 - to 1,000-fold weaker than that of gp120, suggesting that a very particular arrangement 
a

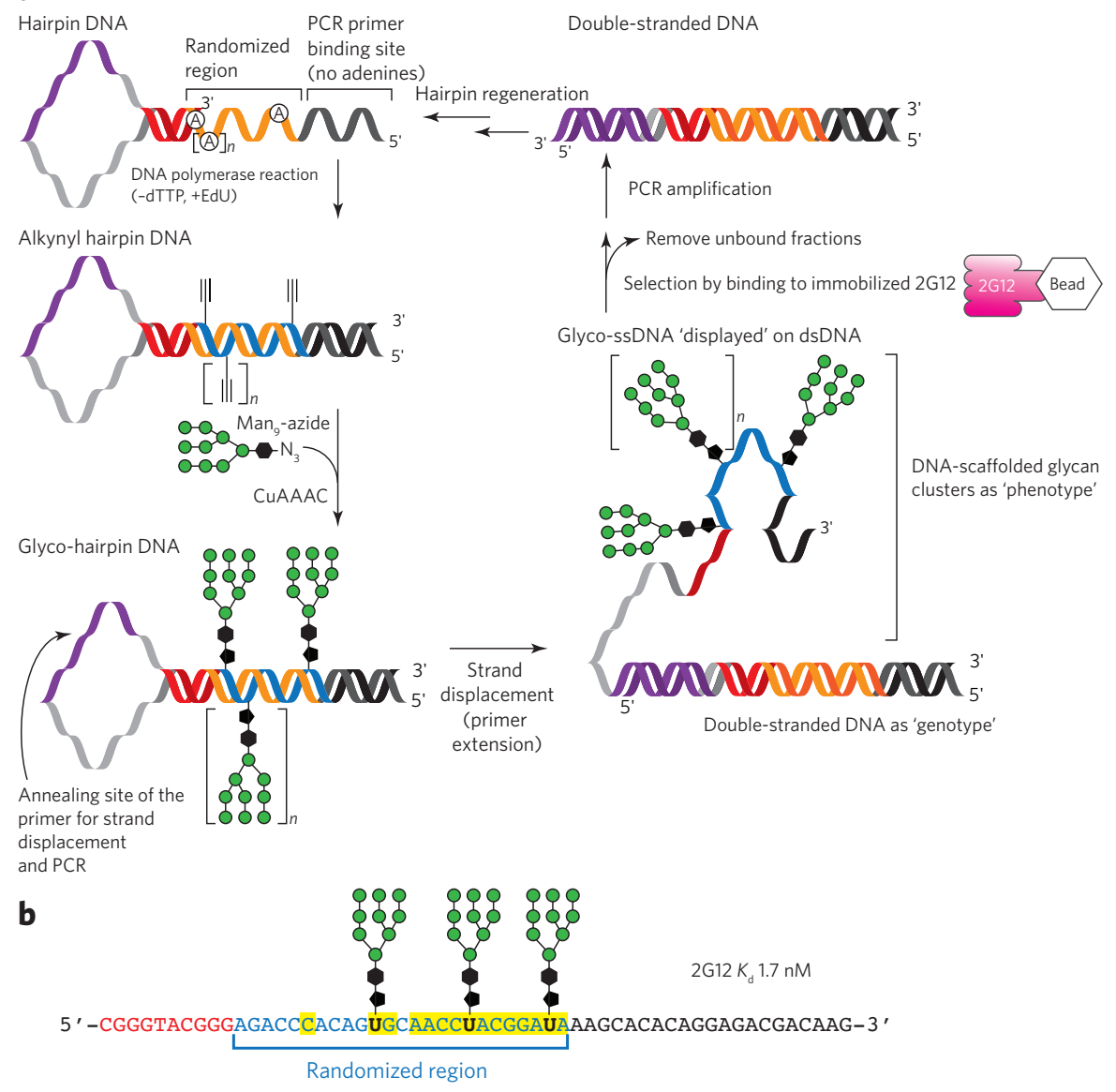

Figure 6 | Directed evolution of DNA-supported glycoclusters antigenic for bnAb $\mathbf{2 G 1 2}$.

(a) Summary of SELMA ${ }^{76,77,79}$. A library of $\sim 10^{13}$ multivalent $\mathrm{Man}_{9}$ presentations is created by using click chemistry to attach glycans to $10^{13}$ alkyne-containing DNA scaffolds. After incubation of the library with $2 \mathrm{G} 12$, sequences that bind are amplified by PCR and subjected to further rounds of click glycosylation and selection until the library converges on highly antigenic structures. EdU, 5-ethynyl-2'-deoxyuridine. (b) A representative glycoDNA obtained from selection, which binds $2 \mathrm{G} 12$ as tightly as does gp120, with a $K_{\mathrm{d}}$ of $1.7 \mathrm{nM}^{79}$. Yellow highlights show the consensus sequence observed in selected clones.

was detected for HIV virus that had been engineered to contain only high-mannose glycans; however, wild-type HIV was not neutralized. It is likely that these antibodies specifically bound to alternative arrangements of high-mannose glycans present only on the artificially overmannosylated virus but not present in the 2 G12 epitope on wildtype virus.

\section{Directed evolution of 2G12 epitope mimics}

The most antigenic 'rationally designed' 2 G12 epitope mimics to date achieve 2 G12 recognition only with a highly multivalent glycan presentation (9-300 copies) ${ }^{67,71}$. Because $2 \mathrm{G} 12$ binds a cluster of just a few (3-4) glycans in its natural target, gp120 (refs.

of high-mannose oligosaccharides is necessary to accurately mimic the epitope of $2 \mathrm{G} 12$. Very high $2 \mathrm{G} 12$ affinity $\left(K_{\mathrm{d}} \sim 3 \mathrm{nM}\right)$ was observed for some glycodendrimer constructs (Fig. $\mathbf{5 f})^{67}$, which is most likely due to a combination of very high multivalency ( 9 or 27 copies of oligomannose glycan) and/or the resultant high density of presentation.

Despite binding to 2G12, none of the above constructs has been reported to elicit antibodies which can bind gp120 or neutralize HIV, although in some cases robust mannose-binding antibody responses were detected ${ }^{57,63,68,70,71}$. Among several possible explanations, the simplest is that none of the tested constructs truly recapitulated a gp120-like presentation of oligomannose residues. In all cases, 2G12 affinities were at least 50-fold weaker than that of the natural gp120-2G12 interaction, or else strong binding was achieved only with a much higher number of glycans than the three or four involved in the 2G12-gp120 interaction. Another possible explanation is that 2G12-like antibodies may be inherently difficult to elicit because the domain-exchanged structure of $2 \mathrm{G} 12$ is exceptionally rare; thus, special adjuvants and carriers or other completely new strategies may be required to achieve the high degree of somatic hypermutation necessary for domain exchange.

Aside from the chemically defined immunogens described above, microbially derived oligomannose constructs deserve special mention. It has been reported that some bacterial surface lipooligosaccharides contain the $\mathrm{Man}_{4}$ motif sufficient for 2G12 recognition, and the immunogenicity of the heat-killed bacteria has been tested in mice ${ }^{73}$. Anti-mannose and anti-gp120 antibodies were elicited, although HIV neutralization was not detected. In another approach, immunogenicity studies were reported with whole yeast cells engineered to express only high-mannose $\left(\mathrm{Man}_{8} \mathrm{GlcNAc}_{2}\right)$ glycans on their surface proteins $s^{74,75}$. The resulting antibody response bound to mannose glycans and to gp 120 protein. In this case, neutralization
$12,15,16)$, we have been interested in achieving a native-strength interaction with constructs bearing a similarly small number of glycans. Our strategy has been to allow highly antigenic oligomannose clusters evolve from very diverse populations of random multivalent arrangements. We have developed two systems for glycocluster evolution in which glycans are positioned on evolving scaffolds composed of either DNAs ${ }^{76,77,79}$ or peptides ${ }^{78}$ (Figs. 6 and 7).

In our DNA-based system (Fig. 6), termed SELMA (selection with modified aptamers $)^{76}$, libraries of $\sim 10^{13}$ DNA scaffolds are generated, in which thymidine is replaced by an alkyne-containing base, ethynyl deoxyuridine. Glycan azides are then used to chemically glycosylate all of the alkynes in the DNA library using copper-assisted alkyne azide cycloaddition (CuAAAC) or 'click' chemistry ${ }^{89,90}$. The glycosylated libraries are incubated with 2 G12 to select for the most antigenic clusterings of the glycan, and the bound fraction is amplified by PCR and reglycosylated to produce the next-generation library for selection. The first selections from $\mathrm{Man}_{4}$-decorated ${ }^{76}$ and $\mathrm{Man}_{9}$-decorated ${ }^{77}$ DNA libraries afforded 150- to 500-nM binders of 2 G12 containing five to ten glycans and having neither the desired low nanomolar affinity nor the desired low multivalency to match that of the native gp120-2G12 interaction. However, markedly improved results were obtained when the temperature of the $2 \mathrm{G} 12$ selection step was increased to $37^{\circ} \mathrm{C}^{79}$. High temperature favored selection of structures that not only had gp120-like (1-15 nM) affinity for 2G12 but also contained just 3-4 glycans.

In further studies ${ }^{78}$, we developed a system for multivalent glycopeptide evolution, combining mRNA display technology with CuAAAC glycosylation (Fig. 7). In mRNA display, a library of peptides is generated by ribosomal translation of mRNA that has been modified so that each peptide is covalently tagged by its encoding mRNA sequence ${ }^{91}$. Translation is done with an unnatural alkynyl amino acid to produce alkynyl peptides, and CuAAAC glycosylation 
converts these to a library of glycopeptides ${ }^{92}$. The most antigenic species are selected for binding to 2G12, their mRNA tags are amplified by RT-PCR, and the library can then be retranslated for the next round of selection. Consistent with our results from the DNA-based SELMA selection method, we found that glycopeptides with low multivalency (3-4 glycans) and very tight 2 G12 binding affinity $(\sim 1 \mathrm{nM})$ were obtained only after high-temperature selection. Although immunogenicity studies have not yet been conducted with these evolved constructs, these directed evolution methods seem to be useful for generating highly antigenic structures and may also be useful for targeting the newer and more broadly neutralizing PG and PGT antibodies, which bind a combination of peptide and glycan elements.

\section{PG9- and PG16-targeted vaccine design}

Until 2009, 2G12 was the only HIV bnAb reported to bind a carbohydrate epitope, and its unique domain-exchanged structure has presented a challenge to vaccine development. Although it has been shown that relatively few substitutions in the germline sequence are necessary to bring about the domain exchangefeature of 2 G12 (ref.87), there is still no evidence that domain-exchanged antibodies can be elicited by vaccination. However, since 2009, high-throughput screening of B-cell clones from 'elite neutralizer' patients has yielded reports of dozens of new glycan-binding monoclonal antibodies, which, unlike 2G12, are not domain exchanged and have the conventional Y/T antibody topology $y^{13,14,19,21,22,26}$. The first new antibodies in this class, PG9 and its close relative PG16, neutralize HIV with much more breadth and potency than 2G12 (ref. 7). For instance, PG9 was shown to neutralize up to $77 \%$ of strains in a 162 -pseudovirus panel (versus $32 \%$ for $2 \mathrm{G} 12$ ), with a median halfmaximum inhibitory concentration of $0.23 \mu \mathrm{g} \mathrm{ml}^{-1}$ (versus $2.4 \mu \mathrm{g}$ $\mathrm{ml}^{-1}$ for $2 \mathrm{G} 12$ ). These newer bnAbs are thus regarded as substantially improved templates for vaccine design.

Design of immunogens that might elicit PG9- or PG16-like antibodies must be informed by knowledge of what structures these bnAbs bind to. PG9 and PG16 bind preferentially to trimeric gp120, and mutation studies show that a PNG site at N160 is required for binding. Moreover, X-ray crystallographic studies of PG9 (ref. 13) in complex with a segment of the gp120 V1/V2 region engrafted onto a scaffold protein showed that PG9 makes extensive contacts with a $\mathrm{Man}_{5} \mathrm{GlcNAc}_{2}$ high-mannose glycan at N160, and lesser contacts with a second glycan at either of two spatially close positions, N156 or N173 (Fig. 8a). Because this $\mathrm{V} 1 / \mathrm{V} 2$ construct was

Figure 7 | Directed evolution of glycopeptides antigenic for bnAb 2G12. (a) Scheme of glycopeptide selection by mRNA display ${ }^{78}$. A library of $\sim 10^{13}$ glycopeptides is created by using click chemistry to attach Man, $_{9}$ glycans to $\sim 10^{13}$ alkyne-containing peptides, each of which is 'barcoded' by covalent attachment to its encoding mRNA using the mRNA display method ${ }^{91}$. The resulting library of $\sim 10^{13}$ glycopeptides is incubated with 2G12, and the bound glycopeptides are amplified by PCR of their attached mRNAs, followed by retranslation of the PCR product and click glycosylation. Selection and amplification is repeated until strong $2 \mathrm{G} 12$ binders are obtained. HPG, homopropargylglycine. (b) A representative selected glycopeptide, which contains four glycans, exhibits a $2 \mathrm{G} 12 K_{\mathrm{d}}$ of $0.97 \mathrm{nM}$, comparable to the affinity observed for gp120 itself.

\section{a} DNA

b produced in $\mathrm{N}$-acetylglucosaminyltransferase I (GnTI)-negative cells, yielding homogenous glycosylation with $\mathrm{Man}_{5} \mathrm{GlcNAc}_{2}$, the $\mathrm{X}$-ray structure does not clarify the glycoform bound at N156 or N173, although the precise observed fit of this N160 glycan into the antibody points to a high likelihood that $\mathrm{Man}_{5} \mathrm{GlcNAc}_{2}$ is the glycoform recognized by PG9 at that position in the native viral trimer. In addition to the interaction with glycans, extensive contacts could be observed with the gp120 V1/V2 polypeptide sequence. Subsequent electron microscopy studies showed that PG9 binds trimeric gp140 at the interface between protomers, near the trimer apex, and provides evidence that a second N160 glycan on a neighboring gp120 protomer may also be involved in PG9 recognition ${ }^{93}$.

Synthetic studies have provided insight into the nature of the glycans recognized by PG9. Recently, chemoenzymatic synthesis methods were used to prepare a panel of divalent V1/V2 glycopeptides to explore this question (Fig. 8b ${ }^{80}$. Glycopeptides containing $\mathrm{Man}_{5} \mathrm{GlcNAc}_{2}$ at position N160 and a sialylated biantennary glycan at N156 or N173 were recognized by PG9 with 20-fold stronger affinity than glycopeptides containing $\mathrm{Man}_{5} \mathrm{GlcNAc}_{2}$ at both positions. Although the observed recognition was in the micromolar range, these studies provided evidence that the PG9 epitope involves a complex glycan at N156 or N173. Additional evidence for the importance of a complex- or hybrid-type glycan came from STD-NMR and crystallographic studies of PG16, showing binding contacts to the sialic acid moiety of both complex- and hybrid-type glycans ${ }^{24}$.

In another study of the PG9 epitope glycopeptides, dimers of synthetic V1/V2 glycopeptides were synthesized, bearing only high-mannose $\left(\mathrm{Man}_{5} \mathrm{GlcNAc}_{2}\right)$ glycans at both N160 and N156 positions (four glycans total) and exhibited relatively tight (30 nM) recognition by PG9 (Fig. 8b) ${ }^{81}$. Although the tighter apparent binding of these constructs to PG9 may be partly due to avidity effects (PG9 IgG was used in these binding studies, whereas PG9 Fab was used with the chemoenzymatically synthesized glycopeptides ${ }^{80}$ ) it is also possibly due to the dimeric construct's mimicry of the quaternary features of the PG9 epitope, where a $\mathrm{Man}_{5} \mathrm{GlcNAc}_{2}$ glycan on each half of the peptide dimer may play the part of the N160 of each gp120 protomer. Alternatively, the increased binding of the dimer may be due to stabilization of its $\beta$-sheet secondary structure, affording a more orderly presentation of either the glycans or the

ORF $\mathrm{P}_{\mathrm{T7}} \longrightarrow \mathrm{SD} \stackrel{\text { (randomized) }}{\text { Constant }}$ 7. PCR ampilification vavavavavaraia (7. PCR ampilification
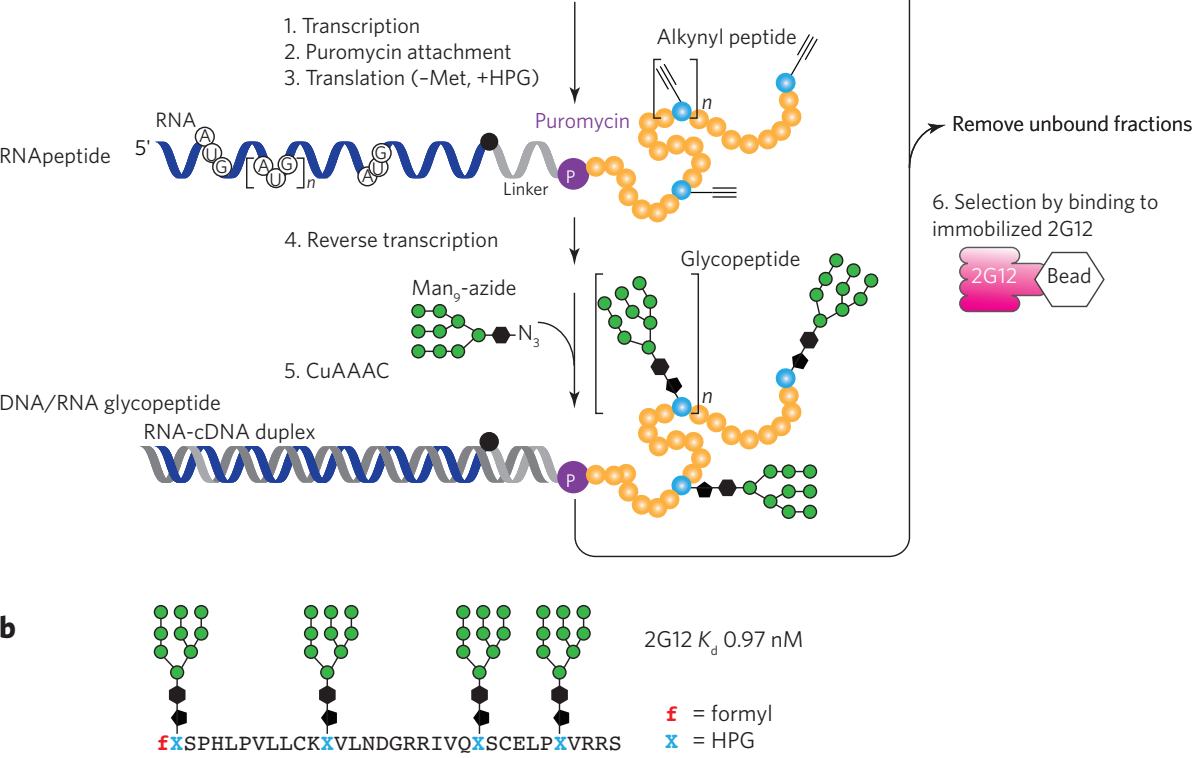
a

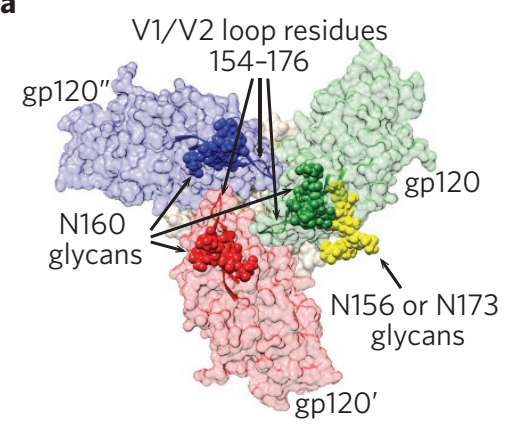

b

Wang's V1/V2 glycopeptides:

(High-mannose at N160, sialylated complex at N156 and N173)

Less antigenic glycoforms:

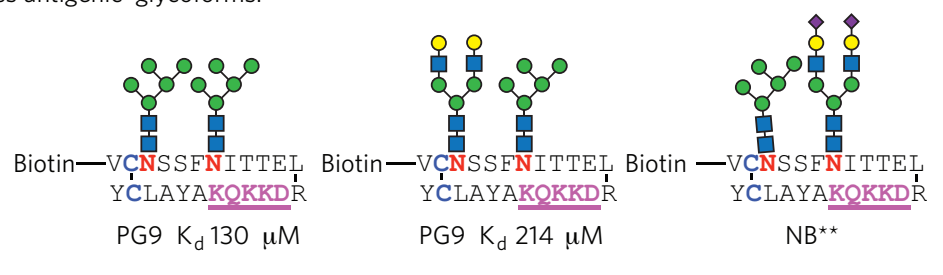

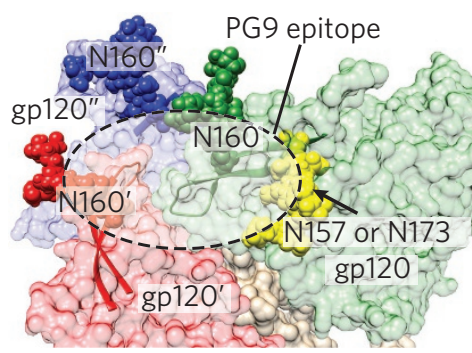

Danishefsky's V1/N2 glycopeptides:

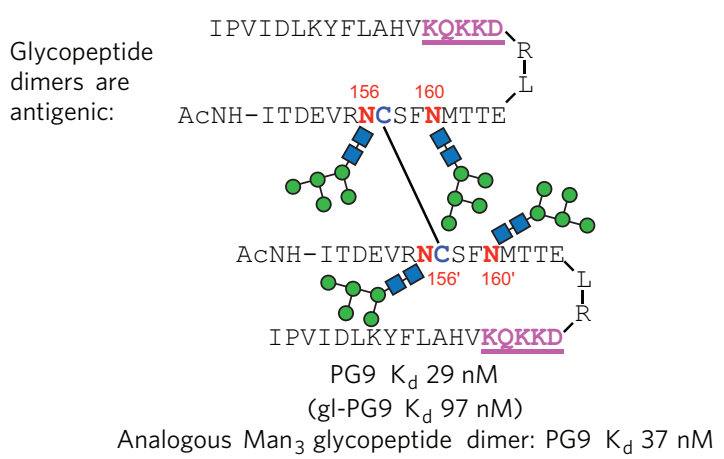

$\mathrm{Man}_{3}$ monomer

glycopeptide not antigenic:

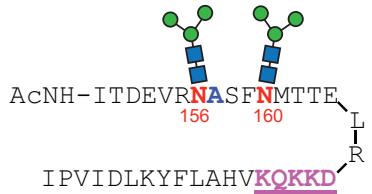

$\mathrm{NB}^{\star}$

Figure 8 | PG9 epitope elements and antigenic glycopeptides. (a) Model of PG9 epitope elements on gp120 trimer. Gp120 protomers shown in red, blue and green, with corresponding N160 glycans for each protomer shown in the same colors near the trimer apex. N156 or N173 glycan on one protomer is depicted in yellow. All other glycans are omitted for clarity. PG9 most likely interacts with high-mannose glycans at N160 of two adjacent gp120

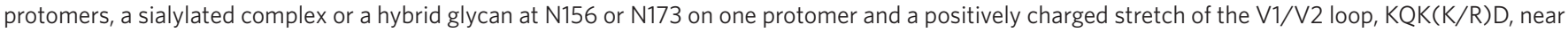

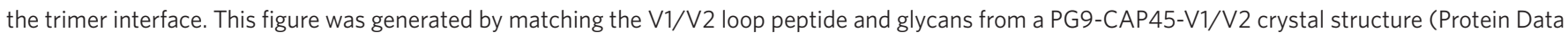
Bank (PDB) code 3U4E) onto the BG505-SOSIP-gp140 trimer crystal structure (PDB code 4NCO). (b) Synthetic glycopeptides containing PG9 epitope

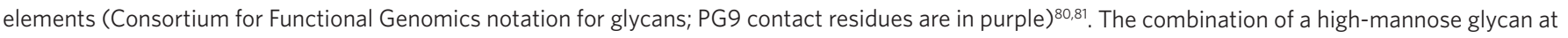
$\mathrm{N} 160$ and a sialylated complex glycan at N156 is most antigenic for monomeric peptide constructs, although dimerization to mimic the quaternary nature of the PG9 epitope and/or stabilize the peptide conformation appears to produce a more antigenic structure even without the complex glycan at N156. $\mathrm{NB}^{\star}$, no binding up to $8 \mu \mathrm{M}$; $\mathrm{NB}^{\star \star}$, no binding up to $100 \mu \mathrm{M}$.

positively charged peptide epitope component (KQKKD). To show that dimerization was essential for antigenicity, the authors demonstrated that a similar $\mathrm{Man}_{3}$ V1/V2 glycopeptide bound PG9 only in the oxidized cysteine-dimerized form. Notably, the dimeric $\mathrm{Man}_{5}$ construct also exhibited $90 \mathrm{nM}$ affinity for the germline precursor of PG9 (gl-PG9). This is important because nearly all gp120 and gp140 constructs so far have failed to bind gl-bnAbs ${ }^{9,35,37,38,82}$. By initially stimulating germline PG9 B cells, this immunogen may be able to begin the antibody maturation pathway from a point that could eventually mature into a PG9-like response.

\section{Deglycosylated immunogens to target germline bnAbs}

Whereas the above approaches aim to elicit antibodies that bind the glycan shield, alternative approaches are directed at the conserved peptide epitopes underneath that shield. One such epitope, the CD4-binding site (CD4 BS), is functionally essential for infection and is therefore the most conserved neutralization epitope within gp120. BnAbs that target this epitope, such as VRC01 and the related NIH45-46, exhibit $\sim 90 \%$ neutralization breadth ${ }^{9,10}$. However, potent VRC01-like antibodies rarely and slowly arise in natural infection, perhaps because the CD4 BS is a recessed epitope or because it is poorly immunogenic. There has been interest in overcoming this problem by tailoring immunogens specifically to bind the germline precursor of VRC01 (gl-VRC01), thereby specifically targeting the antibody development pathway that originally led to VRC01 in the patient from which this antibody was isolated. Interestingly, unmodified Env proteins fail to bind gl-VRC01 (refs. 9,37); however, engineering of deglycosylated Env constructs is a promising approach to address this problem. In one study of trimeric gp140 mutants, it was found that removal the glycan at N276, and to a lesser extent glycans at N460 and N463, restored nanomolar recognition by gl-VRC01 and gl-NIH45-46 (ref. 28). It was shown that the effect of glycan deletion is primarily to increase the on rate in the antibody-protein interaction, as opposed to decreasing the off rate, consistent with removal of a steric obstacle to binding rather than an alteration in the epitope structure. In a related study, N276 deglycosylation, followed by a combination of computational design and directed library screening, identified variants of the highly truncated gp120 fragment 'engineered outer domain' eOD with affinities for gl-VRC01 and gl-NIH45-46 of $44 \mathrm{nM}$ and 
$410 \mathrm{nM}$, respectively ${ }^{27}$. Although these constructs show encouraging antigenicity for gl-bnAbs, animal germline antibodies differ from those of humans, and immunogenicity studies must therefore be conducted either in humans or in mice engineered to express the targeted human germline B cells. Moreover, even if the desired gl-VRC01 response can be initiated, additional strategies may be necessary to bring about affinity maturation toward mature VRC01 specificity. This may include boosting with immunogens designed to stimulate B cells at intermediate stages along the maturation pathway, mimicking the coevolution of virus and antibody that has been observed in nature ${ }^{82}$.

\section{Conclusion and outlook}

2G12, with its unique domain-exchanged structure, was for a long time the only HIV bnAb known to bind carbohydrates, and there was therefore some concern that the glycan shield was too unusual a target to be relevant for HIV vaccine design. With the discovery of more than a dozen additional antibodies of the PG and PGT families, it is now clear not only that glycan epitopes are commonly targeted by the immune system but also that this occurs in most 'elite-neutralizer' patients. Although the newly discovered antibodies, unlike 2G12, all have a conventional Y/T topology, they are nevertheless highly mutated from their germline ancestors. To elicit such unusual antibodies, it may be necessary to target specific germline antibodies and also to use adjuvants or vaccine regimens that maximally drive affinity maturation. Although many of the newer bnAbs exhibit above $70 \%$ neutralization breadth, it will most likely be necessary to design multivalent vaccines targeting several bnAb specificities to achieve a useful breadth of protection. Fortunately, carbohydrate vaccine efforts are supported by continuously improving tools for glycan analysis, glycoengineering of proteins, chemoenzymatic and chemical synthesis and computational and evolution-based glycostructure design.

\section{Received 5 August 2014; accepted 3 October 2014; published} online 13 November 2014

\section{References}

1. Esparza, J. A brief history of the global effort to develop a preventive HIV vaccine. Vaccine 31, 3502-3518 (2013)

This paper is a historical overview of HIV vaccine efforts detailing the several paradigm shifts that have occurred from the 1980s until the present day.

2. Kwong, P.D. \& Mascola, J.R. Human antibodies that neutralize HIV-1: identification, structures, and B cell ontogenies. Immunity 37, 412-425 (2012).

3. Burton, D.R. et al. Efficient neutralization of primary isolates of HIV-1 by a recombinant human monoclonal antibody. Science 266, 1024-1027 (1994).

4. Trkola, A. et al. Human monoclonal antibody 2G12 defines a distinctive neutralization epitope on the gp120 glycoprotein of human immunodeficiency virus type 1. J. Virol. 70, 1100-1108 (1996).

5. Muster, T. et al. Cross-neutralizing activity against divergent human immunodeficiency virus type 1 isolates induced by the gp41 sequence ELDKWAS. J. Virol. 68, 4031-4034 (1994).

6. Stiegler, G. et al. A potent cross-clade neutralizing human monoclonal antibody against a novel epitope on gp41 of human immunodeficiency virus type 1. AIDS Res. Hum. Retrovir. 17, 1757-1765 (2001).

7. Walker, L.M. et al. Broad and potent neutralizing antibodies from an African donor reveal a new HIV-1 vaccine target. Science 326, 285-289 (2009).

8. Corti, D. et al. Analysis of memory B cell responses and isolation of novel monoclonal antibodies with neutralizing breadth from HIV-1-infected individuals. PLoS ONE 5, e8805 (2010).

9. Scheid, J.F. et al. Sequence and structural convergence of broad and potent HIV antibodies that mimic CD4 binding. Science 333, 1633-1637 (2011).

10. Wu, X. et al. Rational design of envelope identifies broadly neutralizing human monoclonal antibodies to HIV-1. Science 329, 856-861 (2010).

11. Calarese, D.A. et al. Dissection of the carbohydrate specificity of the broadly neutralizing anti-HIV-1 antibody 2G12. Proc. Natl. Acad. Sci. USA 102, 13372-13377 (2005).

12. Calarese, D.A. et al. Antibody domain exchange is an immunological solution to carbohydrate cluster recognition. Science 300, 2065-2071 (2003). This paper discloses the crystal structure of bnAb $2 \mathrm{G} 12$ (Fab) ${ }_{2}$ bound to $\mathrm{Man}_{9} \mathrm{GlcNAc}_{2}$, revealing the unique domain-exchanged structure of this antibody, and proposes a model of the gp120-2G12 interaction.
13. McLellan, J.S. et al. Structure of HIV-1 gp120 V1/V2 domain with broadly neutralizing antibody PG9. Nature 480, 336-343 (2011).

This paper illustrates the ability of PG9 to bind a combination of glycan and V1/V2 peptide elements on a gp120-related glycoprotein. Importantly, this paper shows that bnAbs do not have to recognize only peptide or only glycan and that bnAb recognition of carbohydrates is possible without a domain-exchanged antibody structure.

14. Pejchal, R. et al. A potent and broad neutralizing antibody recognizes and penetrates the HIV glycan shield. Science 334, 1097-1103 (2011). This paper shows that an additional class of highly potent bnAbs (PGT) binds both glycan and $\mathrm{V} 3$ peptide elements.

15. Sanders, R.W. et al. The mannose-dependent epitope for neutralizing antibody $2 \mathrm{G} 12$ on human immunodeficiency virus type 1 glycoprotein gp120. J. Virol. 76, 7293-7305 (2002)

16. Scanlan, C.N. et al. The broadly neutralizing anti-human immunodeficiency virus type 1 antibody 2 G12 recognizes a cluster of $\alpha 1 \rightarrow 2$ mannose residues on the outer face of gp120. J. Virol. 76, 7306-7321 (2002).

17. Walker, L.M. et al. A limited number of antibody specificities mediate broad and potent serum neutralization in selected HIV-1 infected individuals. PLoS Pathog. 6, e1001028 (2010).

18. Lavine, C.L. et al. High-mannose glycan-dependent epitopes are frequently targeted in broad neutralizing antibody responses during human immunodeficiency virus type 1 infection. J. Virol. 86, 2153-2164 (2012).

19. Walker, L.M. et al. Broad neutralization coverage of HIV by multiple highly potent antibodies. Nature 477, 466-470 (2011).

This paper reports the discovery of numerous glycan-binding bnAbs of unprecedented potency and shows that numerous such antibodies could be isolated from several individuals, targeting distinct epitopes on gp120.

20. Mouquet, H. et al. Complex-type N-glycan recognition by potent broadly neutralizing HIV antibodies. Proc. Natl. Acad. Sci. USA 109, E3268-E3277 (2012)

21. Kong, L. et al. Supersite of immune vulnerability on the glycosylated face of HIV-1 envelope glycoprotein gp120. Nat. Struct. Mol. Biol. 20, 796-803 (2013).

22. Julien, J.-P. et al. Broadly neutralizing antibody PGT121 allosterically modulates CD4 binding via recognition of the HIV-1 gp120 V3 base and multiple surrounding glycans. PLoS Pathog. 9, e1003342 (2013).

This paper presents evidence that the high neutralization potency of a PGTclass bnAb is due not only to binding but also to an allosteric deactivation of CD4 binding activity.

23. Julien, J.-P. et al. Crystal structure of a soluble cleaved HIV-1 envelope trimer. Science 342, 1477-1483 (2013).

This paper reports the first crystal structure of a gp120-related construct in trimeric form. This structure also includes bound PGT122 Fab, showing for the first time the interaction of a glycan-binding bnAb with a more or less complete gp120 construct, at this resolution.

24. Pancera, M. et al. Structural basis for diverse N-glycan recognition by HIV 1-neutralizing V1-V2-directed antibody PG16. Nat. Struct. Mol. Biol. 20, 804-813 (2013)

25. Falkowska, E. et al. Broadly neutralizing HIV antibodies define a glycandependent epitope on the prefusion conformation of gp 41 on cleaved envelope trimers. Immunity 40, 657-668 (2014).

This paper and the next report the most recent new class of glycan-binding bnAbs, which binds to an epitope near the gp120-gp41 interface.

26. Blattner, C. et al. Structural delineation of a quaternary, cleavage-dependent epitope at the gp41-gp120 interface on intact HIV-1 env trimers. Immunity 40, 669-680 (2014).

27. Jardine, J. et al. Rational HIV immunogen design to target specific germline B cell receptors. Science 340, 711-716 (2013).

28. McGuire, A.T. et al. Engineering HIV envelope protein to activate germline B cell receptors of broadly neutralizing anti-CD4 binding site antibodies. J. Exp. Med. 210, 655-663 (2013).

29. Davis, K.L. et al. High titer HIV-1 V3-specific antibodies with broad reactivity but low neutralizing potency in acute infection and following vaccination. Virology 387, 414-426 (2009)

30. Wei, X. et al. Antibody neutralization and escape by HIV-1. Nature $\mathbf{4 2 2}$, 307-312 (2003).

This paper presents the concept that HIV eludes the immune response in part by masking important epitopes with a glycan shield.

31. Gray, E.S. et al. Neutralizing antibody responses in acute human immunodeficiency virus type 1 subtype c infection. J. Virol. 81, 6187-6196 (2007).

32. Bunnik, E.M., Pisas, L., van Nuenen, A.C. \& Schuitemaker, H. Autologous neutralizing humoral immunity and evolution of the viral envelope in the course of subtype b human immunodeficiency virus type 1 infection. J. Virol. 82, 7932-7941 (2008).

33. Moore, P.L. et al. The C3-V4 region is a major target of autologous neutralizing antibodies in human immunodeficiency virus type 1 subtype $\mathrm{c}$ infection. J. Virol. 82, 1860-1869 (2008)

34. Chen, W. et al. Characterization of germline antibody libraries from human 
umbilical cord blood and selection of monoclonal antibodies to viral envelope glycoproteins: implications for mechanisms of immune evasion and design of vaccine immunogens. Biochem. Biophys. Res. Commun. 417, 1164-1169 (2012)

This paper and the next provide evidence that special efforts may be required to engage germline antibodies with gp120-related constructs, perhaps because HIV has evolved to avoid binding the human germline antibody repertoire.

35. Xiao, X. et al. Germline-like predecessors of broadly neutralizing antibodies lack measurable binding to HIV-1 envelope glycoproteins: implications for evasion of immune responses and design of vaccine immunogens. Biochem. Biophys. Res. Commun. 390, 404-409 (2009).

36. Hoot, S. et al. Recombinant HIV envelope proteins fail to engage germline versions of anti-CD4bs bnAbs. PLoS Pathog. 9, e1003106 (2013).

37. Zhou, T. et al. Structural basis for broad and potent neutralization of HIV-1 by antibody VRC01. Science 329, 811-817 (2010).

38. McGuire, A.T., Glenn, J.A., Lippy, A. \& Stamatatos, L. Diverse recombinant HIV-1 envs fail to activate B cells expressing the germline B cell receptors of the broadly neutralizing anti-HIV-1 antibodies PG9 and 447-52d. J. Virol. 88, 2645-2657 (2014)

39. Earl, P.L., Doms, R.W. \& Moss, B. Oligomeric structure of the human immunodeficiency virus type 1 envelope glycoprotein. Proc. Natl. Acad. Sci. USA 87, 648-652 (1990).

40. Moore, P.L. et al. Nature of nonfunctional envelope proteins on the surface of human immunodeficiency virus type 1. J. Virol. 80, 2515-2528 (2006). This paper describes the phenomenon of 'junk' Env.

41. Sanders, R.W. et al. A next-generation cleaved, soluble HIV-1 env trimer BG505 SOSIP.664 gp140, expresses multiple epitopes for broadly neutralizing but not non-neutralizing antibodies. PLoS Pathog. 9, e1003618 (2013).

42. Zhu, X., Borchers, C., Bienstock, R.J. \& Tomer, K.B. Mass spectrometric characterization of the glycosylation pattern of HIV-gp120 expressed in $\mathrm{CHO}$ cells. Biochemistry 39, 11194-11204 (2000).

43. Mizuochi, T. et al. Carbohydrate structures of the human-immunodeficiencyvirus (HIV) recombinant envelope glycoprotein gp120 produced in Chinese hamster ovary cells. Biochem. J. 254, 599-603 (1988).

44. Go, E.P. et al. Characterization of host-cell line specific glycosylation profiles of early transmitted/founder HIV-1 gp120 envelope proteins. J. Proteome Res. 12, 1223-1234 (2013)

45. Go, E.P. et al. Characterization of glycosylation profiles of HIV-1 transmitted/ founder envelopes by mass spectrometry. J. Virol. 85, 8270-8284 (2011)

46. Go, E.P. et al. Glycosylation site-specific analysis of clade c HIV-1 envelope proteins. J. Proteome Res. 8, 4231-4242 (2009).

47. Go, E.P. et al. Glycosylation site-specific analysis of HIV envelope proteins (JR-FL and CON-S) reveals major differences in glycosylation site occupancy, glycoform profiles, and antigenic epitopes $\bigotimes$ accessibility. J. Proteome Res. 7 , 1660-1674 (2008)

48. Timpe, L.C. et al. Systemic alteration of cell-surface and secreted glycoprotein expression in malignant breast cancer cell lines. Glycobiology 23, 1240-1249 (2013)

49. Apweiler, R., Hermjakob, H. \& Sharon, N. On the frequency of protein glycosylation, as deduced from analysis of the SWISS-PROT database. Biochim. Biophys. Acta 1473, 4-8 (1999).

50. van Liempt, E. et al. Specificity of DC-SIGN for mannose- and fucosecontaining glycans. FEBS Lett. 580, 6123-6131 (2006).

51. Feinberg, H., Castelli, R., Drickamer, K., Seeberger, P.H. \& Weis, W.I. Multiple modes of binding enhance the affinity of DC-SIGN for high mannose N-linked glycans found on viral glycoproteins. J. Biol. Chem. 282, 4202-4209 (2007).

52. van Montfort, T. et al. HIV-1 N-glycan composition governs a balance between dendritic cell-mediated viral transmission and antigen presentation. J. Immunol. 187, 4676-4685 (2011).

53. Moldt, B. et al. Highly potent HIV-specific antibody neutralization in vitro translates into effective protection against mucosal SHIV challenge in vivo. Proc. Natl. Acad. Sci. USA 109, 18921-18925 (2012).

54. Klein, F. et al. HIV therapy by a combination of broadly neutralizing antibodies in humanized mice. Nature 492, 118-122 (2012).

55. Mascola, J.R. et al. Protection of macaques against vaginal transmission of a pathogenic HIV-1/SIV chimeric virus by passive infusion of neutralizing antibodies. Nat. Med. 6, 207-210 (2000).

56. Hessell, A.J. et al. Broadly neutralizing human anti-HIV antibody $2 \mathrm{G} 12$ is effective in protection against mucosal SHIV challenge even at low serum neutralizing titers. PLoS Pathog. 5, e1000433 (2009).

57. Joyce, J.G. et al. An oligosaccharide-based HIV-1 2G12 mimotope vaccine induces carbohydrate-specific antibodies that fail to neutralize HIV-1 virions. Proc. Natl. Acad. Sci. USA 105, 15684-15689 (2008).

58. Krauss, I.J. et al. Fully synthetic carbohydrate HIV antigens designed on the logic of the 2G12 antibody. J. Am. Chem. Soc. 129, 11042-11044 (2007).

59. Wang, J., Li, H., Zou, G. \& Wang, L.X. Novel template-assembled oligosaccharide clusters as epitope mimics for HIV-neutralizing antibody 2G12. Design, synthesis, and antibody binding study. Org. Biomol. Chem. 5, 1529-1540 (2007).
60. Yang, Q., Li, C.S., Wei, Y.D., Huang, W. \& Wang, L.X. Expression, glycoform characterization, and antibody-binding of HIV-1 V3 glycopeptide domain fused with human IgG1-Fc. Bioconjug. Chem. 21, 875-883 (2010).

61. Li, H. et al. Chemoenzymatic synthesis of HIV-1 V3 glycopeptides carrying two $\mathrm{N}$-glycans and effects of glycosylation on the peptide domain. J. Org. Chem. 70, 9990-9996 (2005).

62. Wang, L.X., Ni, J., Singh, S. \& Li, H. Binding of high-mannose-type oligosaccharides and synthetic oligomannose clusters to human antibody $2 \mathrm{G} 12$ Implications for HIV-1 vaccine design. Chem. Biol. 11, 127-134 (2004).

63. Ni, J., Song, H.J., Wang, Y.D., Stamatos, N.M. \& Wang, L.X. Toward a carbohydrate-based HIV-1 vaccine: synthesis and immunological studies of oligomannose-containing glycoconjugates. Bioconjug. Chem. 17, 493-500 (2006).

64. Li, H. \& Wang, L.X. Design and synthesis of a template-assembled oligomannose cluster as an epitope mimic for human HIV-neutralizing antibody 2G12. Org. Biomol. Chem. 2, 483-488 (2004).

65. Gorska, K., Huang, K.-T., Chaloin, O. \& Winssinger, N. DNA-templated homoand heterodimerization of peptide nucleic acid encoded oligosaccharides that mimick the carbohydrate epitope of HIV. Angew. Chem. Int. Ed. Engl. 48 7695-7700 (2009).

66. Ciobanu, M. et al. Selection of a synthetic glycan oligomer from a library of DNA-templated fragments against DC-SIGN and inhibition of HIV gp120 binding to dendritic cells. Chem. Commun. (Camb.) 47, 9321-9323 (2011).

67. Wang, S.K. et al. Targeting the carbohydrates on HIV-1: interaction of oligomannose dendrons with human monoclonal antibody 2G12 and DCSIGN. Proc. Natl. Acad. Sci. USA 105, 3690-3695 (2008)

68. Kabanova, A. et al. Preparation, characterization and immunogenicity of HIV-1 related high-mannose oligosaccharides-CRM197 glycoconjugates. Glycoconj. J. 27, 501-513 (2010).

69. Marradi, M. et al. Gold nanoparticles coated with oligomannosides of HIV-1 glycoprotein gp120 mimic the carbohydrate epitope of antibody $2 \mathrm{G} 12$. J. Mol Biol. 410, 798-810 (2011).

70. Astronomo, R.D. et al. A glycoconjugate antigen based on the recognition motif of a broadly neutralizing human immunodeficiency virus antibody, $2 \mathrm{G} 12$, is immunogenic but elicits antibodies unable to bind to the self glycans of gp120. J. Virol. 82, 6359-6368 (2008).

71. Astronomo, R.D. et al. Defining criteria for oligomannose immunogens for HIV using icosahedral virus capsid scaffolds. Chem. Biol. 17, 357-370 (2010).

72. Doores, K.J. et al. A nonself sugar mimic of the HIV glycan shield shows enhanced antigenicity. Proc. Natl. Acad. Sci. USA 107, 17107-17112 (2010)

73. Clark, B.E. et al. A bacterial lipooligosaccharide that naturally mimics the epitope of the HIV-neutralizing antibody 2 G12 as a template for vaccine design. Chem. Biol. 19, 254-263 (2012).

74. Luallen, R.J. et al. An engineered Saccharomyces cerevisiae strain binds the broadly neutralizing human immunodeficiency virus type 1 antibody 2 G12 and elicits mannose-specific gp120-binding antibodies. J. Virol. 82, 6447-6457 (2008).

75. Agrawal-Gamse, C. et al. Yeast-elicited cross-reactive antibodies to HIV env glycans efficiently neutralize virions expressing exclusively high-mannose N-linked glycans. J. Virol. 85, 470-480 (2011).

76. MacPherson, I.S. et al. Multivalent glycocluster design through directed evolution. Angew. Chem. Int. Ed. Engl. 50, 11238-11242 (2011).

77. Temme, J.S., Drzyzga, M.G., MacPherson, I.S. \& Krauss, I.J. Directed evolution of 2G12-targeted nonamannose glycoclusters by SELMA. Chemistry 19, 17291-17295 (2013).

78. Horiya, S., Bailey, J.K., Temme, J.S., Guillen Schlippe, Y.V. \& Krauss, I.J. Directed evolution of multivalent glycopeptides tightly recognized by HIV antibody 2G12. J. Am. Chem. Soc. 136, 5407-5415 (2014).

This paper describes the use of glycopeptide-directed evolution to produce highly antigenic $2 \mathrm{G} 12$ epitope mimic glycopeptides.

79. Temme, J.S., MacPherson, I.S., DeCourcey, J.F. \& Krauss, I.J. High temperature SELMA: evolution of DNA-supported oligomannose clusters which are tightly recognized by HIV bnAb 2G12. J. Am. Chem. Soc. 136, 1726-1729 (2014). This paper describes the use of glycoDNA-directed evolution (SELMA) to produce highly antigenic 2 G12 epitope mimic glycoclusters scaffolded by DNA.

80. Amin, M.N. et al. Synthetic glycopeptides reveal the glycan specificity of HIVneutralizing antibodies. Nat. Chem. Biol. 9, 521-526 (2013). This paper uses chemoenzymatic synthesis methods to produce a panel of glycopeptides used to interrogate the glycan specificity of PG9 and PG16.

81. Alam, S.M. et al. Recognition of synthetic glycopeptides by HIV-1 broadly neutralizing antibodies and their unmutated ancestors. Proc. Natl. Acad. Sci. USA 110, 18214-18219 (2013). This paper reports the most antigenic PG9 epitope mimic glycopeptide synthesized to date and shows that it is also recognized well by germline PG9.

82. Liao, H.-X. et al. Co-evolution of a broadly neutralizing HIV-1 antibody and founder virus. Nature 496, 469-476 (2013).

83. Bonomelli, C. et al. The glycan shield of HIV is predominantly oligomannose independently of production system or viral clade. PLoS ONE 6, e23521 (2011). This paper and the next show the differences in glycan content between recombinant gp120 monomer and gp120 trimer from viral particles. 
84. Doores, K.J. et al. Envelope glycans of immunodeficiency virions are almost entirely oligomannose antigens. Proc. Natl. Acad. Sci. USA 107, 13800-13805 (2010).

85. Buchacher, A. et al. Generation of human monoclonal antibodies against HIV1 proteins; electrofusion and Epstein-Barr virus transformation for peripheral blood lymphocyte immortalization. AIDS Res. Hum. Retrovir. 10, 359-369 (1994).

86. Doores, K.J., Fulton, Z., Huber, M., Wilson, I.A. \& Burton, D.R. Antibody 2G12 recognizes di-mannose equivalently in domain- and nondomain-exchanged forms but only binds the HIV-1 glycan shield if domain exchanged. J. Virol. 84, 10690-10699 (2010).

87. Huber, M. et al. Very few substitutions in a germ line antibody are required to initiate significant domain exchange. J. Virol. 84, 10700-10707 (2010).

88. Wang, L.-X. Synthetic carbohydrate antigens for HIV vaccine design. Curr. Opin. Chem. Biol. 17, 997-1005 (2013).

89. Rostovtsev, V.V., Green, L.G., Fokin, V.V. \& Sharpless, K.B. A stepwise Huisgen cycloaddition process: copper(I)-catalyzed regioselective 'ligation' of azides and terminal alkynes. Angew. Chem. Int. Ed. Engl. 41, 2596-2599 (2002).

90. Kolb, H.C., Finn, M.G. \& Sharpless, K.B. Click chemistry: diverse chemical function from a few good reactions. Angew. Chem. Int. Ed. Engl. 40, 20042021 (2001).

91. Liu, R.H., Barrick, J.E., Szostak, J.W. \& Roberts, R.W. Optimized synthesis of RNA-protein fusions for in vitro protein selection. Methods Enzymol. 318, 268-293 (2000).

92. Josephson, K., Hartman, M.C.T. \& Szostak, J.W. Ribosomal synthesis of unnatural peptides. J. Am. Chem. Soc. 127, 11727-11735 (2005).

93. Julien, J.P. et al. Asymmetric recognition of the HIV-1 trimer by broadly neutralizing antibody PG9. Proc. Natl. Acad. Sci. USA 110, 4351-4356 (2013). This paper reports cryo-EM data showing that PG9 binds the apex of the gp120 trimer at the interface of two gp120 protomers.

94. Fennie, C. \& Lasky, L.A. Model for intracellular folding of the human immunodeficiency virus type 1 gp120. J. Virol. 63, 639-646 (1989).
95. Li, Y., Luo, L., Thomas, D.Y. \& Kang, C.Y. The HIV-1 env protein signal sequence retards its cleavage and down-regulates the glycoprotein folding. Virology 272, 417-428 (2000).

96. Willey, R.L., Bonifacino, J.S., Potts, B.J., Martin, M.A. \& Klausner, R.D. Biosynthesis, cleavage, and degradation of the human immunodeficiency virus 1 envelope glycoprotein gp160. Proc. Natl. Acad. Sci. USA 85, 9580-9584 (1988).

97. Decroly, E. et al. The convertases furin and pcl can both cleave the human immunodeficiency virus (HIV)-1 envelope glycoprotein gp160 into gp120 (HIV-1 SU) and gp41 (HIV-1 TM). J. Biol. Chem. 269, 12240-12247 (1994).

98. Fenouillet, E. \& Gluckman, J.-C. Immunological analysis of human immunodeficiency virus type 1 envelope glycoprotein proteolytic cleavage. Virology 187, 825-828 (1992).

99. Hallenberger, S. et al. Inhibition of furin-mediated cleavage activation of HIV-1 glycoprotein gpl60. Nature 360, 358-361 (1992).

100. Stein, B.S. \& Engleman, E.G. Intracellular processing of the gp160 HIV1 envelope precursor. Endoproteolytic cleavage occurs in a cis or medial compartment of the Golgi complex. J. Biol. Chem. 265, 2640-2649 (1990).

\section{Acknowledgments}

The authors gratefully acknowledge the support of the US National Institutes of Health (R01 AI090745 and R01 AI113737).

\section{Competing financial interests}

The authors declare competing financial interests: details accompany the online version of the paper.

\section{Additional information}

Reprints and permissions information is available online at http://www.nature.com/ reprints/index.html. Correspondence and requests for materials should be addressed to I.J.K. 